\title{
RETRIEVAL OF ATMOSPHERIC BACKSCATTER AND EXTINCTION PROFILES WITH THE ALADIN AIRBORNE DEMONSTRATOR (A2D)
}

\author{
Alexander Geiss ${ }^{1,}$, Uwe Marksteiner ${ }^{2}$, Oliver Lux ${ }^{2}$, Christian Lemmerz ${ }^{2}$, Oliver \\ Reitebuch $^{2}$, Thomas Kanitz ${ }^{1}$ and Anne Grete Straume-Lindner ${ }^{1}$ \\ ${ }^{1}$ European Space Agency (ESA-ESTEC), 2200 AG Noordwijk, The Netherlands, \\ *alexander.geiss@esa.int \\ ${ }^{2}$ German Aerospace Center (DLR), Institute of Atmospheric Physics, Oberpfaffenhofen, 82234 \\ Wessling, Germany
}

\begin{abstract}
By the end of 2017, the European Space Agency (ESA) will launch the Atmospheric laser Doppler instrument (ALADIN), a direct detection Doppler wind lidar operating at $355 \mathrm{~nm}$. An important tool for the validation and optimization of ALADIN's hardware and data processors for wind retrievals with real atmospheric signals is the ALADIN airborne demonstrator A2D. In order to be able to validate and test aerosol retrieval algorithms from ALADIN, an algorithm for the retrieval of atmospheric backscatter and extinction profiles from A2D is necessary.

The A2D is utilizing a direct detection scheme by using a dual Fabry-Pérot interferometer to measure molecular Rayleigh signals and a Fizeau interferometer to measure aerosol Mie returns. Signals are captured by accumulation charge coupled devices (ACCD). These specifications make different steps in the signal preprocessing necessary.

In this paper, the required steps to retrieve aerosol optical products, i. e. particle backscatter coefficient $\beta_{p}$, particle extinction coefficient $\alpha_{p}$ and lidar ratio $S_{p}$ from A2D raw signals are described.
\end{abstract}

\section{INTRODUCTION}

The Atmospheric Dynamics Mission Aeolus (ADM-Aeolus) $[1,2]$ is ESA's second Earth Explorer core mission, with a date of launch by the end of 2017. The direct detection Doppler wind lidar ALADIN carried by ADM-Aeolus emits laser pulses at an ultraviolet wavelength of $355 \mathrm{~nm}$. The mission's first objective is to obtain global observations of horizontally projected line-of-sight wind profiles with regard to testing the capability for future operational wind satellites and the aim to improve numerical weather prediction [3] and climate studies. However, as being the first high spectral resolution lidar (HSRL) in space, ALADIN allows also an accurate vertical profiling of aerosol and cloud optical properties on a global scale as a secondary product. An important tool for the development, testing, validation and optimization of the hardware and data processors of ALADIN, is the ALADIN airborne demonstrator A2D $[4,5]$. With the same fundamental concepts and design but slightly different specifications, it can be used to validate and demonstrate the capabilities of ALADIN. The German aerospace centre operated A2D from ground and from an airborne platform during campaigns in 2006, 2007, 2009, 2015 and 2016. Valuable information about the instrument characteristic, calibration and data processing were gained with realistic atmospheric signals and provided important recommendations for ALADIN. However, with focusing on the retrieval of wind profiles, no aerosol optical properties were retrieved with atmospheric measurements. In order to validate and to optimize the aerosol retrieval for ALADIN [6] by using atmospheric measurements, a retrieval of particle backscatter and extinction profiles for A2D must be developed. 
Table 1: Key parameters of A2D.

\begin{tabular}{ll}
\hline Platform & Airborne or ground \\
LOS pointing & $20^{\circ}$ \\
Laser & Nd:YAG \\
Wavelength & $354.89 \mathrm{~nm}$ \\
Laser line width & $45 \mathrm{Mhz}$ \\
Pump system & diode \\
Pulse repetition rate & $50 \mathrm{~Hz}$ \\
Pulse duration & $25 \mathrm{~ns}$ \\
Pulse energy & $50-60 \mathrm{~mJ}$ \\
Laser divergence & $90 \mu \mathrm{rad}$ for $\pm 3 \sigma$ \\
Laser beam diameter & $0.016 \mathrm{~m}$ \\
Telescope diameter & $0.2 \mathrm{~m}$ \\
Configuration & Coaxial \\
Field of View & $100 \mu \mathrm{rad}$ \\
Mie receiver & Fizeau \\
Rayleigh receiver & Fabry-Pérots \\
ACCD detector & \\
$\quad$ Imaging zone & $16 \times 16$ pixel \\
$\quad$ Memory zone & $25 \times 16$ pixel \\
Altitude range & $0-10 \mathrm{~km}$ \\
Vertical resolution & $296-2370 \mathrm{~m}$ \\
Min. horizontal & $80 \mathrm{~m}$ \\
resolution & \\
\hline
\end{tabular}

\section{METHODOLOGY}

\subsection{A2D - principle of operation}

The A2D is based on a diode-pumped frequency-tripled Nd:YAG laser emitting pulses with an energy of 50-60 mJ with a repetition frequency of $50 \mathrm{~Hz}$ (see Table 1). The outgoing laser light is circularly polarized and the laser beam is steered via a piezo-electrically controlled mirror. Its position is tracked with an UV-camera and a CCD (see Fig. 1). A telescope with $0.2 \mathrm{~m}$ diameter collects the backscattered light. The structural design with a secondary mirror and the additional use of an electro-optical modulator to suppress the near-field signal results in a region of incomplete overlap up to a distance of $2000 \mathrm{~m}$ from the lidar [5]. Although receiving crossand parallel-polarized backscattered light only the latter is measured and conducted towards a combination of a Fizeau interferometer to detect the narrowband aerosol return and a dual Fabry-Pérot interferometer to measure the

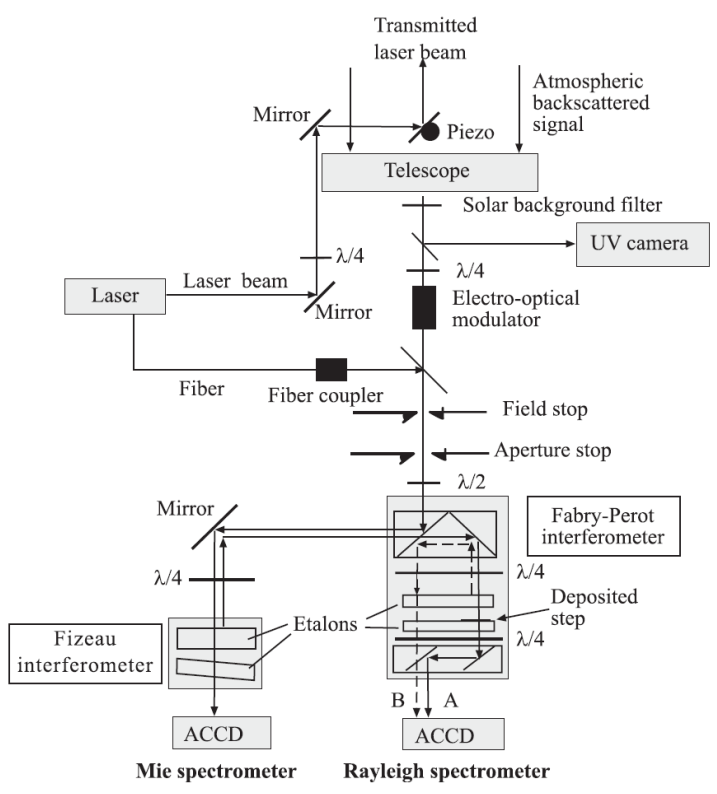

Figure 1: Illustration of the optical configuration of A2D [4].

broadband molecular signals. ACCDs are used to capture the signals resulting in 25 available range bins from which 20 are atmospheric range bins. As a consequence, the vertical resolution is limited to $296-2370 \mathrm{~m}$ depending on the integration length of the signal. With a measurement summed up over 18 laser pulses and a flight speed of $200 \mathrm{~m} / \mathrm{s}$, a minimum horizontal resolution of $80 \mathrm{~m}$ is achieved.

\subsection{Signal pre-processing}

Before an aerosol retrieval can be applied to A2D data, different signal corrections are necessary to obtain pure Rayleigh and Mie signals which can be further processed. At first, a so-called detection chain offset and the background signal must be subtracted from each range bin for both channels. The variable range bin length requires a scaling of the signal intensities of each layer depending on their integration length. The next and fundamental step is a cross-talk correction caused by an incomplete decoupling of both receiver channels. As a consequence, the Mie channel collects Rayleigh 


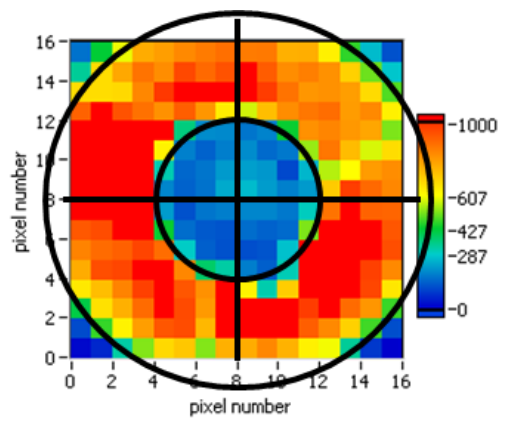

Figure 2: Rayleigh background on the Mie channel in imaging mode. Blue colors in the center are related to the secondary mirror obscuration. Auxiliary lines are marked in black. Courtesy $O$. Reitebuch (DLR).

signals and the Rayleigh channel is influenced by Mie returns. In the first case, due to the imaging characteristic of the Mie spectrometer (MSP), the optical beam path from the telescope to the MSP is imaged on the Mie ACCD. Thus, an obscuration in the beam path from the secondary mirror and its spider is present on the Mie ACCD as shown in Fig. 2 and requires a so called telescope obscuration (TOBS) correction. For ALADIN the obscuration for every single pixel is derived from an optical model. A constant factor for each pixel is used for the correction. For the A2D, however, a constant TOBS correction is not possible since the illumination of the telescope from the atmospheric signal and the optical path through the A2D is varying. An A2D approach is based on shifting the Mie signal fringe out of the useful spectral range of the Mie receiver (MOUSR) by changing the laser frequency. The remaining telescope image can be monitored and subsequently subtracted from the measurements. The cross-talk contribution from the Mie signal to the Rayleigh channel is corrected according to a method described for ALADIN which is based on using the Mie channel signals [7]. Further corrections for obtaining correct overall Rayleigh intensities must consider the wind induced shift of the Rayleigh-Doppler backscatter spectrum as well as the temperature induced
Rayleigh line broadening.

In order to increase the signal to noise ratio, temporal averaging of the pre-processed signal is needed. Because of the coarse resolution of the A2D, a feature mask is required to avoid averaging over different types of particles (e. g. clouds, elevated aerosol, planetary boundary layer). The reliable determination of the ground return for every measured profile is realized by using a digital elevation model (DEM) based on data from the Shuttle Radar Topography Mission (SRTM) [8] with 3 arcsec resolution. To distinguish between different features, i. e. clouds and aerosol, a segmentation algorithm is combined with threshold values in the signal and gradient of the signal.

\subsection{Aerosol optical products from A2D}

With A2D being a single wavelength high spectral resolution lidar, the independent retrieval of profiles of particle backscatter coefficients $\beta_{p}$, particle extinction coefficients $\alpha_{p}$ and lidar ratios $S_{p}$ is possible if an HSRL-retrieval is applied $[11,12]$. In case of using only the Mie channel, the Klett-Fernald algorithm $[9,10]$ allows a determination of $\beta_{p}$ only. With data from different flight campaigns being available, especially cases with blown snow at the East coast of Greenland and two flights in the Mediterranean region provide sufficient aerosol backscatter for an aerosol retrieval. Preliminary results of the Mie signal after applying the TOBS correction are shown in Fig. 3 for a flight along the Greenland east coast.

\subsection{Validation}

In order to verify the accuracy of retrieved aerosol optical products from $\mathrm{A} 2 \mathrm{D}$, a validation with independent measurements of co-located lidar systems is necessary. To quantify the influence of detecting only parallel-polarized light on the aerosol retrieval, a comparison with a depolarization lidar is needed. Particularly, in 


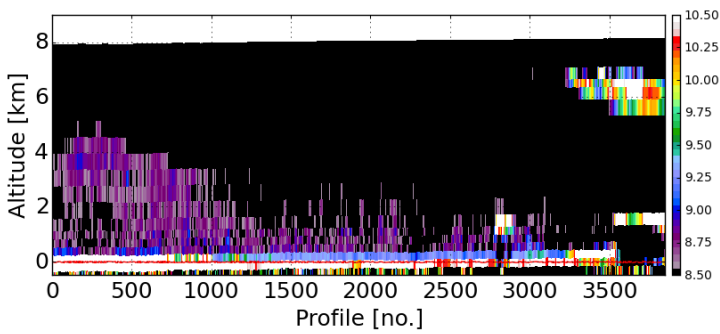

Figure 3: Time height cross section of the A2D Mie signal after TOBS correction (logarithmic scale, a.u.) during flight on 19 May 2015. White colors indicate clouds and violet colors indicate aerosol. The ground is marked as red line.

case of particles with large depolarization ratios (e.g. desert dust, volcanic ash) the determined $\beta_{p}$ from A2D can be considerably smaller than the total particle backscatter coefficient. To test the reliability of the feature mask and results from the Klett-Fernald retrieval, data from calibrated ceilometer networks [13] and additional information from e. g. sun photometer measurements can be used.

\section{CONCLUSIONS}

With A2D having the same fundamental characteristics as ALADIN it is the ideal instrument to perform calibration and validation activities [14] before and after launch of ADM-Aeolus. However, additional steps for a proper aerosol retrieval applicable to A2D and its principle of operation are necessary in order to be able to validate and optimize aerosol products from ALADIN.

\section{References}

[1] A. Stoffelen et al., 2005: The Atmospheric Dynamics Mission for global wind field measurements, Bull. Am. Meteor. Soc. 86, 73-87.

[2] European Space Agency, 1999: Atmospheric Dynamics Mission, Report for Mission Selection, ESA-SP 1233(4), ESTEC, Noordwijk, The Netherlands.

[3] O. Reitebuch, M. Weissmann, 2008: Impact of Airborne and Future Spaceborne Wind Lidar Observations of ADM-Aeolus on Weather
Prediction Skills, 24th Int. Laser and Radar Conference (ILRC 2008), Boulder, Colorado, 23-27.

[4] O. Reitebuch et al., 2009: The Airborne Demonstrator for the Direct-Detection Doppler Wind Lidar ALADIN on ADMAeolus. Part I:Instrument Design and Comparison to Satellite Instrument, J. Atmos. Oceanic Tech. 26 (12), 2516-2530.

[5] U. Paffrath et al., 2008: The Airborne Demonstrator for the Direct-Detection Doppler Wind Lidar ALADIN on ADM-Aeolus. Part II: Simulations and Rayleigh Receiver Radiometric Performance, J. Atmos. Oceanic Tech. 26 (12), 2501-2515.

[6] P. Flamant et al., 2008: ADM-Aeolus retrieval algorithms for aerosol and cloud products, Tellus 60A (2), 273-288.

[7] A. Ansmann et al., 2007: Particle backscatter and extinction profiling with the spaceborne high-spectral-resolution Doppler lidar ALADIN: methodology and simulations, $A p$ plied Optics 46 (26), 6606-6622.

[8] T. G. Farr et al., 2007: The Shuttle Radar Topography Mission, Rev. Geophys. 45, RG2004.

[9] E. W. Eloranta, 2005: High spectral resolution lidar, LIDAR, C. Weitkamp Springer, 2005 143-163.

[10] A. Ansmann and D. Müller, 2005: Lidar and atmospheric aerosol particles, LIDAR, $C$. Weitkamp Springer, 2005 104-141.

[11] J. D. Klett, 1981: Stable analytical inversion solution for processing lidar returns, Appl. Opt. 20 211-220.

[12] F. G. Fernald, 1984: Analysis of atmospheric lidar observations: some comments Appl. Opt. 23 652-653.

[13] M. Wiegner et al., 2014: What is the benefit of ceilometers for aerosol remote sensing? An answer from EARLINET Atmos. Meas. Tech. 7 1979-2014.

[14] A. G. Straume et al., 2016: ESA's spaceborne lidar mission ADM-Aeolus; recent achievements and preparations for launch, The 27th International Laser Radar Conference (ILRC 27), New York City, USA, EPJ Web of Conferences, 119 (2016) 01001. 